\title{
Myoclonus and Cerebellar Ataxia Associated with SARS-CoV-2 Infection: Case Report and Review of the Literature
}

\author{
Nadia Mariagrazia Giannantoni ${ }^{1}$, Elia Rigamonti ${ }^{1}$, Francesca Irene Rampolli ${ }^{1}$, Lorenzo Grazioli-Gauthier ${ }^{1}$, Gilles Allali ${ }^{2,3}$, Gianluca Vanini $^{1}$ \\ ${ }^{1}$ Department of Internal Medicine, Ospedale Regionale di Lugano, Switzerland \\ ${ }^{2}$ Department of Clinical Neurosciences, Division of Neurology, Geneva University Hospitals and Faculty of Medicine, University of Geneva, Switzerland \\ ${ }^{3}$ Department of Neurology, Division of Cognitive and Motor Aging, Albert Einstein College of Medicine, Yeshiva University, Bronx, NY, USA
}

\section{Doi: 10.12890/2021_002531- European Journal of Case Reports in Internal Medicine - ๑ EFIM 2021}

Received: 29/03/2021

Accepted: $16 / 04 / 2021$

Published: 06/05/2021

How to cite this article: Giannantoni NM, Rigamonti E, Rampolli FI, Grazioli-Gauthier L, Allali G, Vanini G. Myoclonus and cerebellar ataxia associated with SARS-CoV-2 infection: case report and review of the literature. EJCRIM 2021;8: doi:10.12890/2021_002531.

Conflicts of Interests: The Authors declare that there are no competing interests.

This article is licensed under a Commons Attribution Non-Commercial 4.0 License

\section{ABSTRACT}

The current SARS-CoV-2/COVID-19 pandemic has led to a global health crisis. The clinical spectrum of SARS-CoV-2 infection ranges from asymptomatic infection to critical illness affecting almost every organ including the central and peripheral nervous systems. Myoclonus, a less expected and relatively unusual neurological complication, together with ataxia, has lately been associated with SARS-CoV-2 infection. We describe the case of a 67-year-old male patient, admitted to our hospital for interstitial bilateral pneumonia due to SARSCoV-2 infection, who progressively developed general myoclonus and later cerebellar ataxia and gait disturbance. Given the timeline from COVID-19 systemic symptoms to neurological manifestations and the normal results of extensive and non-conclusive diagnostic work-up (blood test, lumbar puncture, EEG, cerebral MRI), a para-infectious encephalopathy related to SARS-CoV-2 was contemplated and a high dose of methylprednisolone was started with prompt symptom improvement.

Further investigation and neuroepidemiological studies are needed to help define the mechanism of neuroinvasion and the entire spectrum of neurological manifestations of SARS-CoV-2 infection, even in mildly affected patients, in terms of prevention, treatment and possible neurological sequelae.

\section{LEARNING POINTS}

- SARS-CoV-2 infection can be related to neurological symptoms and sequelae.

- Myoclonus, specifically when associated with ataxia, might represent the expression of COVID-19-related encephalopathy.

- Myoclonus associated with SARS-CoV-2 infection mostly responds to treatment with steroids.

\section{KEYWORDS}

SARS-CoV-2 infection, myoclonus, ataxia, differential diagnoses

\section{INTRODUCTION}

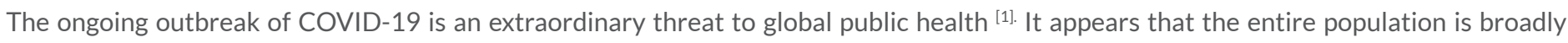
vulnerable to COVID-19, with adults with an average age of 55 years being mostly affected ${ }^{[2]}$. The predominant clinical presentation is respiratory, but nearly every organ system is susceptible to the virus, resulting in several extra-pulmonary manifestations and emerging compelling neurological complications ${ }^{[3]}$. Indeed, recent data demonstrate that SARS-CoV-2 infection may affect the central nervous system (e.g., headache, confusion, pyramidal syndromes, focal neurological deficits, dizziness, cerebellar ataxia, visual disturbance), the 
peripheral nervous system (e.g., impaired eye movement, anosmia and ageusia, trigeminal neuropathy, facial palsy, limb weakness) and muscle (e.g., myalgia, myasthenic crisis, rhabdomyolysis). These neurological manifestations can occur before, during and even after the onset of COVID-19 symptoms ${ }^{[4-6]}$. Generalized myoclonus, a less expected and relatively unusual neurological presentation, has emerged as a consequence of COVID-19 ${ }^{[7-9]}$.

Here, we report an infrequent case of myoclonus associated with ataxia in moderate SARS-CoV-2 illness, and provide an up-to-date review of the associated literature.

\section{CASE DESCRIPTION}

We present the case of a 67-year-old male patient, known for ischaemic heart disease, permanent atrial fibrillation and ethyltoxic liver disease, who was admitted to our hospital for interstitial bilateral pneumonia in the context of a positive nasopharyngeal swab sample for SARSCoV-2. The patient received low-flow $\mathrm{O} 2$ therapy and dexamethasone for 8 days and progressively recovered from respiratory symptoms.

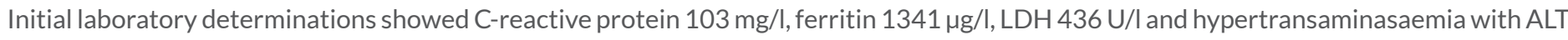
$330 \mathrm{U} / \mathrm{I}$. The rest of biochemistry parameters were normal (Table 1), including the dosage of carbohydrate-deficient transferrin.

Eleven days after the first appearance of respiratory and systemic symptoms (fever, dyspnoea and cough, without anosmia or dysgeusia), the patient progressively developed gait disturbance and disabling and diffuse tremors, requiring a new hospitalization.

Clinically, we observed a mild dysarthria, voice, head and postural tremors, multifocal myoclonic jerks, partially action and stimulus induced, over the limbs, the trunk and the face, which were chaotic, sometimes synchronized and of variable duration, together with limb hypotonus, ocular saccadic movement, gait instability, dysmetria of limbs and postural and gait ataxia. No cloudiness of consciousness, pyramidal or extrapyramidal signs, focal neurological deficits or sensorimotor deficits were noticed during the neurological examination.

Investigations, differential diagnoses and treatment

Several differential diagnoses were considered. The initial presentation was mild and was considered to be a side effect of dexamethasone therapy. As the clinical picture worsened over days, alternative possible diagnoses were considered, including essential tremor, for which the patient received propranolol, and delirium tremens in the context of alcohol withdrawal, treated with oxazepam and vitamin B1 supplementation, without amelioration. Drug-induced myoclonus was excluded considering the absence of favouring medications, such as antipsychotics and anti-depressants, opioids and NMDA agonists. A functional disorder was ruled out after repetitive observations and a psychiatric evaluation.

A broad diagnostic work-up was performed. Contrast cerebral MRI with T2* and DWI sequences showed the absence of acute stroke or microbleeds or a contrast-enhanced lesion in the context of mild cortical atrophy and moderate and diffuse leukoencephalopathy. An extensive biochemical assay revealed normal electrolytes, renal, thyroid and parathyroid function, and negative serology for HIV, Borrelia burgdorferi and Treponema pallidum (Table 1). Cerebrospinal fluid analysis revealed negative SARS-CoV-2 PCR-RT and the absence of pleocytosis, hyperproteinorrachia and intrathecal synthesis. The serological and cerebrospinal search for onconeural antibodies was negative. EEG was performed and resulted normal, without evidence of triphasic waves or epileptic abnormalities.

We hypothesized a para-infectious encephalopathy related to SARS-CoV-2, given the timeline of neurological presentation, corresponding to the resolution of the inflammatory phase of COVID-19 infection, and the absence of other possible explanations (metabolic, iatrogenic or hypoxic). Therefore, we started a therapeutic trial with high-dose intravenous methylprednisolone ( $1 \mathrm{~g}$ for 5 days), followed by a tapering dose of prednisone for 10 days, and initiated symptomatic treatment with clonazepam and levetiracetam. Myoclonic tremor, ataxia and gait instability improved within 14 days of high-dose steroid treatment, with no clinical relapses.

\section{DISCUSSION}

We have described a case of subacute generalized myoclonus associated with ataxia, which was sensitive to high-dose steroids, presumably due to inflammatory encephalopathy related to COVID-19.

In contrast with other case reports and clinical series of steroid-sensitive encephalopathy, cited in the most recent literature, our patient did not present any alteration of vigilance and did not need intensive care support, but similarly to the cases previously described, no structural abnormality on brain MRI was found and no evidence of SARS-CoV-2 presence in the CSF sample was observed, possibly as a result of transient or undetectable virus in the CSF $\left.{ }^{[10,11}\right]$.

The respiratory manifestations of SARS-CoV-2 are well documented and known, but most recently, neurological complications are being increasingly recognized, reported in both mild and severe cases of COVID-19, which appear to be protean and range from alteration of consciousness to myoclonus, with a low incidence of more severe neurological sequelae ${ }^{[5,6,8]}$. 


\section{of Case Reports in \\ Internal Medicine}

\begin{tabular}{|l|l|}
\hline \multicolumn{2}{|l|}{ Basic laboratory findings on arrival } \\
\hline White cell count per $\mathrm{mm}^{3}$ & 3.3 \\
\hline Platelet count per $\mathrm{mm}^{3}$ & 238 \\
\hline Haemoglobin, g/l & 14.5 \\
\hline Lactate dehydrogenase, U/I & 435 \\
\hline Creatinine kinase, U/I & 87 \\
\hline C-reactive protein, $\mathrm{mg} / \mathrm{I}$ & 103 \\
\hline Serum ferritin ng/ml & 1341 \\
\hline D-dimer, ng/ml & Negative (<150 ng/ml) \\
\hline Blood ions (Na, Cl, K, Ca, Mg, P) & Normal \\
\hline Creatinine, $\mu \mathrm{mol} / \mathrm{I}$ & 70 \\
\hline Urea mmol/I & 5.5 \\
\hline Thyroid function & Normal \\
\hline $\begin{array}{c}\text { Serologies } \\
\text { CSF analyses }\end{array}$ & $\begin{array}{l}\text { HIV, Borrelia burgdorferi } \\
\text { and syphilis negative }\end{array}$ \\
\hline $\begin{array}{l}\text { Basic biochemistry and white cell } \\
\text { count in CSF }\end{array}$ & Normal \\
\hline $\begin{array}{l}\text { COVID-19 PCR, IgG, IgM } \\
\text { Onconeural antibodies (liquor and } \\
\text { serum) }\end{array}$ & Negative \\
\hline
\end{tabular}

\begin{tabular}{|c|c|}
\hline \multicolumn{2}{|l|}{ Imaging } \\
\hline $\begin{array}{l}\text { Brain MRI scan (with or without } \\
\text { gadolinium) }\end{array}$ & $\begin{array}{l}\text { Cortical atrophy without } \\
\text { specific pattern and } \\
\text { moderate diffuse } \\
\text { leukoencephalopathy }\end{array}$ \\
\hline Chest CT scan & $\begin{array}{l}\text { Bilateral peripheral } \\
\text { infiltrates }\end{array}$ \\
\hline EEG & Normal \\
\hline \multicolumn{2}{|l|}{ Medication } \\
\hline \multirow[t]{7}{*}{ Previous chronic medication } & Rivaroxaban \\
\hline & Atenolol \\
\hline & Amlodipine \\
\hline & Torasemide \\
\hline & Allopurinol \\
\hline & Simvastatin \\
\hline & Zolpidem \\
\hline
\end{tabular}

Neurological features of SARS-CoV-2 infection mostly manifest within 3 weeksfrom respiratory or systemic symptom onset, are multifactorial and attributed (a) to the virus neurotropism, accomplished by vascular, transcribrial and/or neuronal retrograde dissemination, (b) to an autoimmune response, by means of molecular mimicry and cytokine storming, and finally (c) to multiorgan dysfunction, through mainly cardiorespiratory and metabolic impairment ${ }^{[4-6]}$.

Neurotropism of SARS-CoV-2 is supported by the high prevalence of ageusia and anosmia, often the presenting symptoms of the disease, and the widespread expression of the ACE-2 receptor in the central nervous system, mainly in thalamic nuclei, cerebellum and inferior olivary nuclei, surprisingly with a paucity of reported cases of movement disorders ${ }^{[4,12,13]}$.

Myoclonus is a frequently observed hyperkinetic movement disorder, defined as a sudden, lightening-like muscle jerk due to a plethora of aetiologies and pathophysiological mechanisms ${ }^{[14]}$. Myoclonus observed in patients with COVID-19 may reflect a combination of multiple contributing factors, including metabolic derangement, hypoxia, medication toxicity, and direct or para-infectious complications ${ }^{\text {[7-9, 15] }}$.

We have described a moderately affected SARS-CoV-2 patient presenting with subacute, progressive cortical-subcortical myoclonus (unfortunately not better characterized by EMG) and cerebellar impairment, which ameliorated within 2 weeks of high doses of pulse corticosteroids, clonazepam and levetiracetam.

We searched in PubMed and the Cochrane Library using the terms 'myoclonus', 'ataxia' and 'COVID-19' or 'SARS-CoV-2' and found six clinical cases reported in the literature (Table 2). All published cases involved male patients, as in our case, presenting a mean time of onset of myoclonus and ataxia after the first manifestations of COVID-19 of 18 days (SD \pm 10.4 ) and a mean age of 53.2 years (SD \pm 10.6 ). Moreover, all of the patients underwent brain MRI, with no significant pathological findings reported, but did not undergo an EEG, in contrast to our patient, in whom it was normal ${ }^{[7-9,16]}$. Four of the six patients described in the literature received intravenous immunoglobulin and/or steroids with clinical improvement.

It should be noted that, differently from the cases reported in literature until now, our patient was older and moderately affected by SARSCoV-2 respiratory manifestations, presenting with a slowly progressive neurological syndrome which was promptly sensitive to steroids. In our case, just like the majority of previously described reports, the timing of neurological presentation, the rate of clinical progression, the positive response to anti-inflammatory agents and the absence of clinical recurrence after interruption of steroid therapy, suggest a likely inflammatory-mediated para-infectious or post-infectious mechanism (Table 2). 


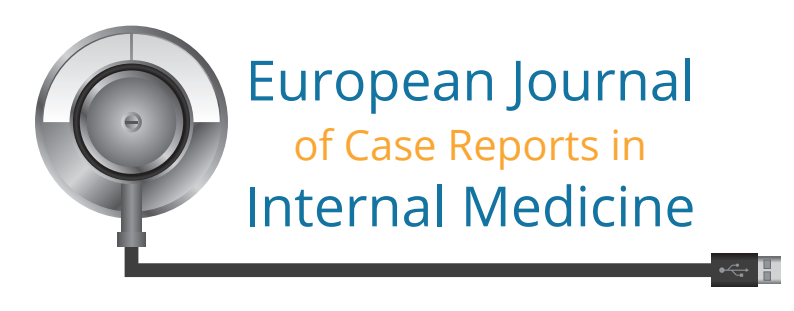

\begin{tabular}{|c|c|c|c|c|c|c|c|}
\hline 邑 & $\begin{array}{l}\frac{0}{0} \\
\frac{\pi}{0} \\
\frac{\pi}{\pi} \\
\frac{\pi}{0} \\
\stackrel{0}{0} \\
z\end{array}$ & 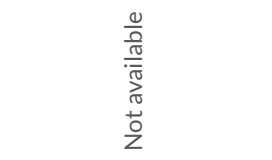 & $\begin{array}{l}\frac{0}{0} \\
\frac{\pi}{\frac{\pi}{\pi}} \\
\frac{\pi}{\pi} \\
\stackrel{0}{0} \\
\frac{0}{z}\end{array}$ & $\begin{array}{l}\frac{0}{0} \\
\frac{0}{0} \\
\frac{\pi}{\sqrt{0}} \\
\frac{\pi}{2} \\
\frac{0}{2}\end{array}$ & $\begin{array}{l}\frac{0}{0} \\
\frac{\pi}{00} \\
\frac{\pi}{\pi 0} \\
\frac{\pi}{0} \\
\frac{0}{2}\end{array}$ & $\begin{array}{l}\frac{0}{0} \\
\frac{0}{0} \\
\frac{\pi}{\pi / 0} \\
\frac{\pi}{0} \\
\frac{0}{2}\end{array}$ & $\begin{array}{l}\overline{\widetilde{\sigma}} \\
\xi_{0} \\
z\end{array}$ \\
\hline 嵌 & $\begin{array}{l}\overline{\widetilde{\sigma}} \\
\sum_{\overline{0}}^{\circ} \\
z\end{array}$ & $\begin{array}{l}\overline{\bar{g}} \\
\sum_{0} \\
\text { z }\end{array}$ & $\begin{array}{l}\overline{\bar{\sigma}} \\
\sum_{\overline{0}} \\
\bar{z}\end{array}$ & $\begin{array}{l}\overline{\bar{\sigma}} \\
\sum_{\overline{0}} \\
z\end{array}$ & $\begin{array}{l}\overline{\bar{\sigma}} \\
\sum_{\overline{0}} \\
z\end{array}$ & $\begin{array}{l}\overline{\bar{\sigma}} \\
\sum_{\bar{g}} \\
z\end{array}$ & $\begin{array}{l}\overline{\bar{\sigma}} \\
\bar{\xi} \\
\bar{z}\end{array}$ \\
\hline 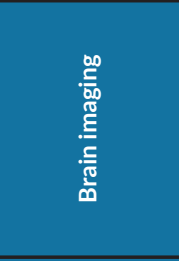 & 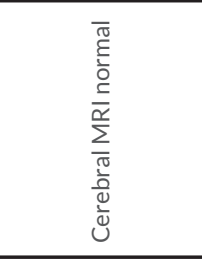 & 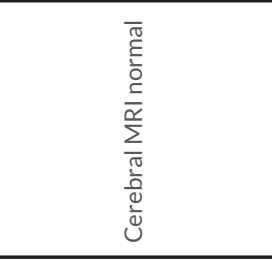 & 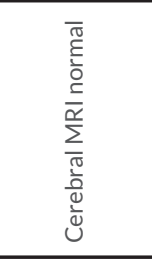 & 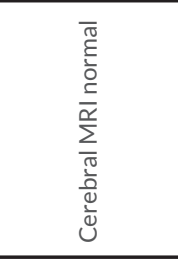 & 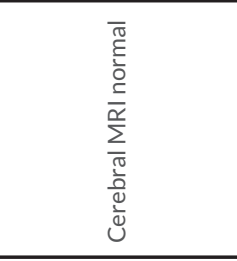 & 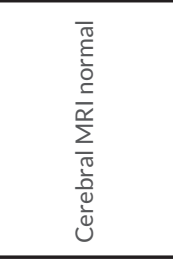 & 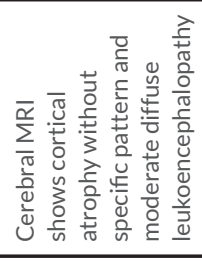 \\
\hline 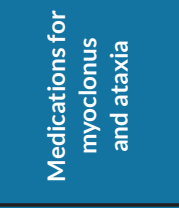 & 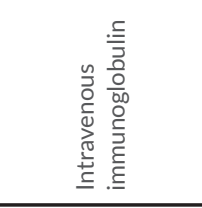 & 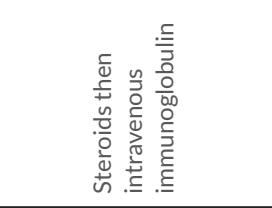 & 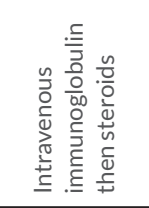 & 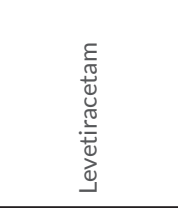 & 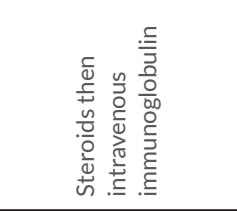 & 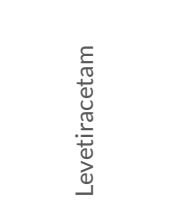 & 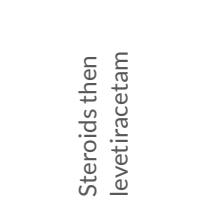 \\
\hline 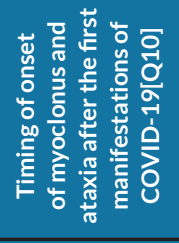 & $\begin{array}{l}\frac{n}{8} \\
\sum_{0}^{\infty} \\
0\end{array}$ & 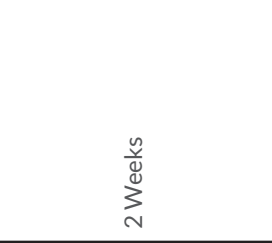 & 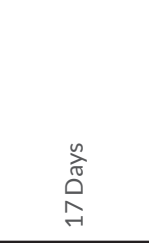 & 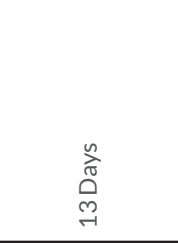 & 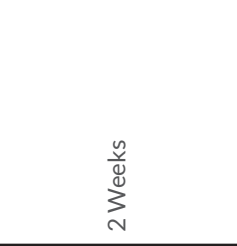 & 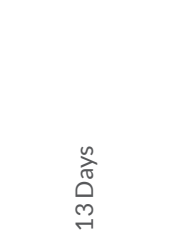 & 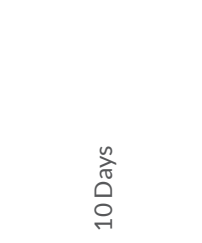 \\
\hline 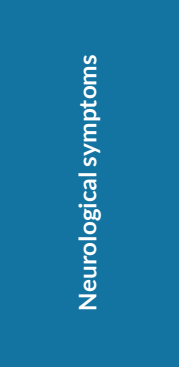 & 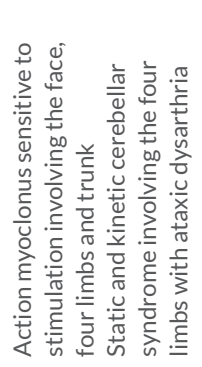 & 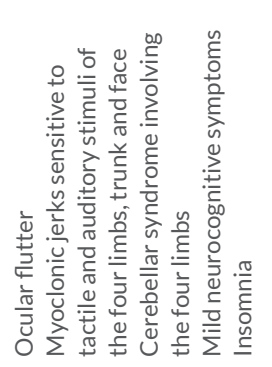 & 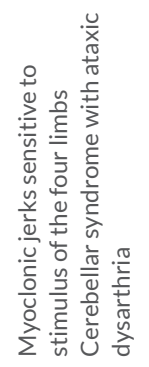 & 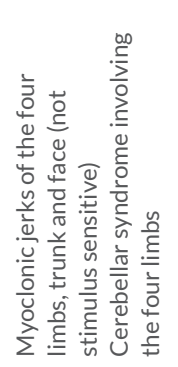 & 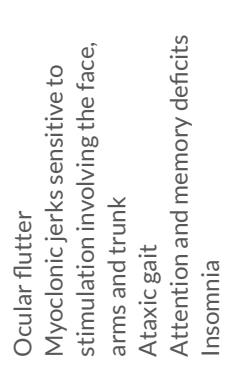 & 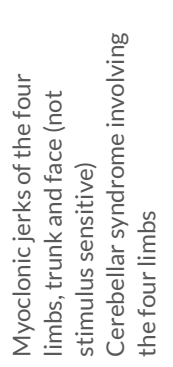 & 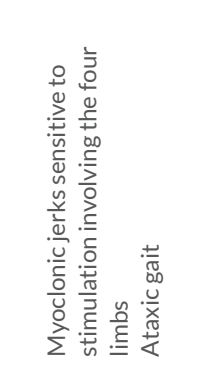 \\
\hline 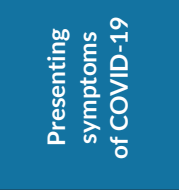 & $\begin{array}{l}\frac{0}{0} \\
\frac{0}{0} \\
\frac{\pi}{\pi} \\
\frac{\pi}{\pi} \\
\stackrel{0}{z} \\
z\end{array}$ & $\begin{array}{l}\frac{0}{0} \\
\frac{0}{0} \\
\frac{\pi}{\pi} \\
\frac{\pi}{\pi} \\
\stackrel{0}{2} \\
z\end{array}$ & $\begin{array}{l}\frac{0}{0} \\
\frac{\pi}{0 \pi} \\
\frac{\pi}{\pi} \\
\frac{\pi}{0} \\
\frac{0}{z}\end{array}$ & $\begin{array}{l}\frac{0}{0} \\
\frac{0}{0} \\
\frac{\pi}{\pi} \\
\frac{\pi}{\pi} \\
\frac{0}{2} \\
z\end{array}$ & 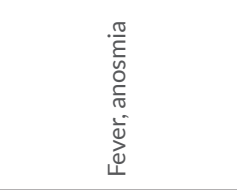 & 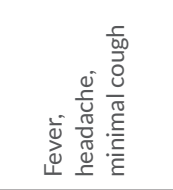 & 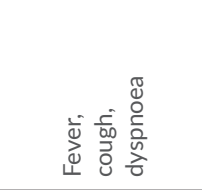 \\
\hline 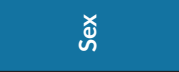 & $\Sigma$ & $\Sigma$ & $\Sigma$ & $\Sigma$ & $\Sigma$ & $\Sigma$ & $\Sigma$ \\
\hline 离 & శె & \& & $\stackrel{N}{ }$ & $\stackrel{\infty}{+}$ & 寸 & $\stackrel{\infty}{+}$ & ก \\
\hline $\begin{array}{l}\text { tั0 } \\
\frac{0}{0} \\
\stackrel{\alpha}{\alpha}\end{array}$ & 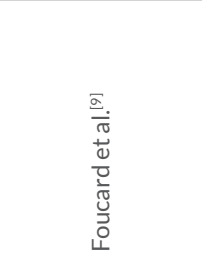 & 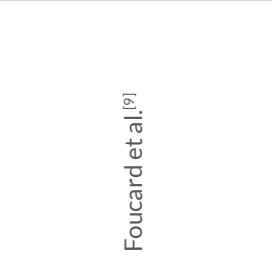 & 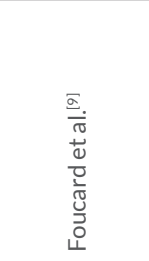 & 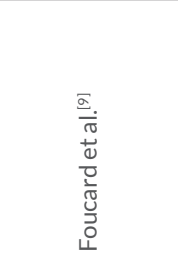 & 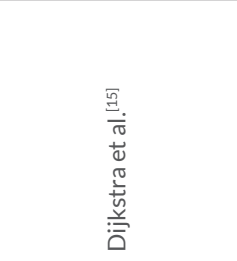 & 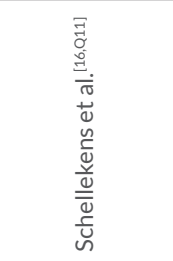 & 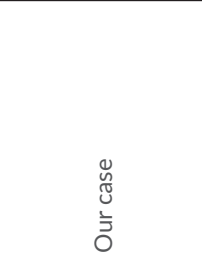 \\
\hline$\dot{z}$ & - & N & $m$ & t & in & م & $\wedge$ \\
\hline
\end{tabular}

Table 2. Characteristics of patients with myoclonus and ataxia with coronavirus disease 2019 
Several differential diagnoses were taken into account before deciding on a clinical reversible scenario probably related to SAR-CoV-2 infection.

An extensive and normal laboratory and instrumental evaluation excluded potential structural or metabolic causes for our clinical presentation. Delirium tremens was ruled out by the progression of myoclonus, the absence of sudden anamnestic alcohol cessation and the clinical absence of other supporting features (fluctuation of attention, dysautonomic failure, sleep-wake disturbances and/or hallucinations). Essential tremor was dismissed because there was no previous history of familial tremor, combined with the lack of response to betablocker and the absence of provocative factors. Finally, a psychogenic disorder was excluded given the presence of objective and nonimitable neurological signs (e.g., hypotonia with Holmes signs) and the shortage of supporting clinical hallmarks (e.g., lack of inconsistency and suggestibility during neurological examination).

\section{CONCLUSIONS}

The mechanism underlying the now evident neuroinvolvement of SARS-CoV-2 is yet not fully understood and further research is essential to help prevent and treat possible neurological sequelae.

By now, emerging and increasingly plentiful clinical data are pointing to a possible relationship between movement disorders and SARSCoV-2 infection, even in moderately affected patients, revealing a wide range of possible manifestations of inflammatory encephalopathy related to COVID-19 that the clinician should bear in mind in the management of these patients.

Neuroepidemiological studies and international registries are needed to help define the full neurological spectrum of SARS-CoV-2 disease and its possible clinical burden, even in mildly affected patients, considering the possible neurodegenerative sequelae of virus exposure.

\section{REFERENCES}

1. Hu B, Guo H, Zhou P, Shi ZL. Characteristics of SARS-CoV-2 and COVID-19. Nat Rev Microbiol 2021;19(3):141-154. doi: 10.1038/s41579-020-00459-7.

2. Wu Z, McGoogan JM. Characteristics of and important lessons from the coronavirus disease 2019 (COVID-19) outbreak in China: summary of a report of 72314 cases from the Chinese Center for Disease Control and Prevention. JAMA 2020;323:1239-1242.

3. Gupta A, Madhavan MV, Sehgal K, Nair N, Mahajan S, Sehrawat TS, et al. Extrapulmonary manifestations of COVID-19. Nat Med 2020;26:1017-1032.

4. Ellul MA, Benjamin L, Singh B, Lant S, Michael BD, Easton A, et al. Neurological associations of COVID-19. Lancet Neurol 2020;19:767-783.

5. Paterson RW, Brown RL, Benjamin L, Nortley R, Wiethoff S, Bharucha T, et al. The emerging spectrum of COVID-19 neurology: clinical, radiological and laboratory findings. Brain 2020;143:3104-3120.

6. Sharifian-Dorche M, Huot P, Osherov M, Wen D, Saveriano A, Giacomini PS, et al. Neurological complications of coronavirus infection; a comparative review and lessons learned during the COVID-19 pandemic. J Neurol Sci 2020;417:117085.

7. Anand P, Zakaria A, Benameur K, Ong C, Putman M, O'Shea S, et al. Myoclonus in patients with coronavirus disease 2019: a multicenter case series. Crit Care Med 2020;48(11):1664-1669.

8. Latorre A, Rothwell JC. Myoclonus and COVID-19: a challenge for the present, a lesson for the future. Mov Dis Clin Pract 2020;7(8):888-890.

9. Foucard C, San-Galli A, Tarrano C, Chaumont H, Lannuzel A, Roze E. Acute cerebellar ataxia and myoclonus with or without opsoclonus: a para-infectious syndrome associated with COVID-19. Eur J Neurol 2021 Jan 25;10.1111/ene.14726. doi: 10.1111/ene.14726.

10. Pilotto A, Odolini S, Masciocchi S, Comelli A, Volonghi I, Gazzina S, et al. Steroid-responsive encephalitis in Covid-19 disease. Ann Neurol 2020;88(2):423-427. doi: 10.1002/ ana.25783.

11. Pugin D, Vargas MI, Thieffry C, Schibler M, Grosgurin O, Pugin J, et al. COVID-19-related encephalopathy responsive to high-dose glucocorticoids. Neurology 2020;95(12):543546.

12. Allen AM, O'Callaghan E, Mendelsohn F, Chai S. Neuronal angiotensin. In: Squire LR, editor. Encyclopedia of neuroscience. New York: Academic Press; p. 697-702.

13. Geyer HL, Kaufman DM, Parihar RK, Mehler MF. Movement disorders in COVID-19: whither art thou? Tremor Other Hyperkinet Mov (N Y) $2020 ; 10: 25$.

14. Eberhardt O, Topka H. Myoclonic disorders. Brain Sci 2017;7(8):103.

15. Dijkstra F, Van den Bossche T, Willekens B, Cras P, Crosiers D. Myoclonus and cerebellar ataxia following COVID-19. Mov Dis Clin Pract 2020;7(8):974-976.

16. Schellekens MMI, Bleeker-Rovers CP, Keurlings PAJ, Mummery CJ, Bloem BR. Reversible Myoclonus-Ataxia as a Postinfectious Manifestation of COVID-19. Mov Dis Clin Pract. 2020;7(8):977-979. 